ARTICLE

DOI: $10.1038 / \mathrm{s} 41467-018-05803-3$

\title{
Runx-dependent and silencer-independent repression of a maturation enhancer in the $C d 4$ gene
}

\author{
Satoshi Kojo (10 ${ }^{1}$, Nighat Yasmin ${ }^{1,2}$, Sawako Muroi ${ }^{1}$, Mari Tenno ${ }^{1}$ \& Ichiro Taniuchi ${ }^{1}$
}

An intronic silencer, $S 4$, in the $C d 4$ gene has been shown to be responsible for the helperlineage-specific expression of CD4; $S 4$ requires Runx complex binding to exert its silencer function against the enhancer-mediated $C d 4$ activation by modulating the epigenetic state of the $C d 4$ gene. Here we identify a late-acting maturation enhancer. Bcl11b plays essential roles for activation of both the early-acting proximal enhancer and maturation enhancer of $\mathrm{Cd} 4$. Notably, Runx complexes suppress these enhancers by distinct mechanisms. Whereas repression of the proximal enhancer depends on the 54 silencer, the maturation enhancer is repressed by Runx in the absence of S4. Moreover, ThPOK, known to antagonize S4-mediated $\mathrm{Cd} 4$ repression, assists Runx complexes to restrain maturation enhancer activation. Distinct modes of $S 4$ silencer action upon distinct enhancers thus unravel a pathway that restricts CD4 expression to helper-lineage cells by silencer-independent and Runx-dependent repression of maturation enhancer activity in cytotoxic-lineage cells.

\footnotetext{
${ }^{1}$ Laboratory for Transcriptional Regulation, RIKEN Center for Integrative Medical Sciences (IMS), 1-7-22 Suehiro-cho, Tsurumi-ku, Yokohama 230-0045, Japan. ${ }^{2}$ Present address: Department of Biomedical Sciences, King Edward Medical University Nelagumbad Mayo Hospital Road, Lahore 54000, Pakistan. Correspondence and requests for materials should be addressed to I.T. (email: ichiro.taniuchi@riken.jp)
} 
C D4 and CD8 glycoproteins function as a co-receptor that assists T-cell antigen receptor (TCR) to recognize antigenic peptide presented by major histocompatibility complex (MHC) class II and class I molecules, respectively ${ }^{1}$. In addition, CD4/CD8 molecules serve as useful markers to define thymocyte developmental stages and helper-lineage and cytotoxic-lineage $\mathrm{T}$ cells ${ }^{2}$. Signals from pre-TCR complexes in $\mathrm{CD}^{-}{ }^{-} \mathrm{CD} 8^{-}$double-negative (DN) thymocyte progenitors induce both $\mathrm{CD} 4$ and $\mathrm{CD} 8$ expression, resulting in the generation of $\mathrm{CD}^{+}{ }^{+} \mathrm{CD} 8{ }^{+}$double-positive (DP) precursor thymocytes. A limited numbers of DP thymocytes, which have passed a process known as positive selection, differentiate further into mature thymocytes ${ }^{3}$. Post-selection thymocytes expressing MHC-class I (MHC-I) restricted TCRs are specified to differentiate into the cytotoxic-lineage and acquire $\mathrm{CD} 4^{-} \mathrm{CD} 8^{+}$single-positive (SP) phenotype by terminating CD4 expression, whereas MHC-class II (MHC-II)-mediated TCR engagement generates $\mathrm{CD}^{+}{ }^{+} \mathrm{CD} 8^{-} \mathrm{SP}$ thymocytes committed to the helper-lineage by inhibiting CD8 expression.

Such stage-specific and lineage-specific expression of CD4/CD8 co-receptors is regulated at the transcriptional level by a combinational regulation of cis-regulatory elements ${ }^{2}$. For instance, insertion of a $434 \mathrm{bp}$ intronic transcriptional silencer (S4) into a transgene together with a $430 \mathrm{bp}$ proximal enhancer $(E 4 p)$ and a minimal $C d 4$ promoter $(P 4)$ is necessary to recapitulate stagespecific and lineage-specific $C d 4$ expression in reporter transgene expression $^{4,5}$. CD4 de-repression from $\mathrm{CD}^{+} \mathrm{T}$ cells upon ablation of the $S 4$ sequences ${ }^{6,7}$. These observations established a model that the single $S 4$ silencer controls helper-lineage specific expression of the $C d 4$ gene $^{8}$. Sequential studies further revealed that binding of Runx transcription factor complexes to $S 4$ through their recognition of two Runx-motifs is essential for $S 4$ activity $^{9,10}$.

Ablation of the E4p from the murine $C d 4$ locus $\left(C d 4^{\Delta E 4 p / \Delta E 4 p}\right.$ mice) also confirmed that $E 4 p$ is essential to initiate $C d 4$ activation $^{11}$. However, despite severely diminished CD4 expression on precursor thymocytes, a small but significant proportion of precursors was positively selected and differentiated into mature thymocytes expressing CD 4 at a lower level in $C d 4^{\Delta E 4 p / \Delta E 4 p}$ mice, leading to an assumption that additional enhancer(s), referred to as a maturation enhancer $(E 4 m)$, should be present ${ }^{11}$. It has also been shown that stage-specific DNA de-methylation in helperlineage cells and continuous DNA methylation in cytotoxiclineage cells are involved in establishment of the heritable active and silent status, and requires E4p and $S 4$ activity, respectively ${ }^{11,12}$. Thus, $C d 4$ gene regulation has served as an ideal model to study how stage-specific and lineage-specific epigenetic modifications are regulated by cis-regulatory elements. However, the genomic region containing $E 4 m$ activity remains elusive, as does the mechanism by which $E 4 m$ activity is regulated.

In this study, we identify the $E 4 m$, validated its role in regulating CD4 expression, and isolate $\mathrm{Bcl11}$ b as an important activator for $E 4 m$. We also show that Runx complexes repress the E4m activity in $\mathrm{CD}^{+} \mathrm{T}$ cells even in the absence of the $S 4$ and find unexpected ThPOK function that prevents premature $E 4 m$ activation by assisting Runx-mediated $E 4 m$ repression. Collectively, our results reveal that Runx complexes repress two enhancers, $E 4 p$ and $E 4 m$, in distinct manners, providing a novel insight that revises a silencer-based model for a lineage-specific Cd4 expression.

\section{Results}

Rescue of E4p function by a heterologous enhancer. It was shown that E4p is necessary for DNA de-methylation of the $C d 4$ gene $^{12}$. To examine whether the activity that induces DNA de- methylation in the $C d 4$ locus is specific to E4p, we tested how a heterologous enhancer behaves in the $C d 4$ locus. Two enhancers, a thymic enhancer $(T E)$ and a proximal enhancer $(P E)$, are present in the Thpok gene encoding the CD4-specific transcription factor ThPOK ${ }^{13,14}$. Low expression of Thpok upon removal of Tet family proteins that are essential for DNA de-methylation ${ }^{15}$ suggests an involvement of DNA de-methylation in activation of the Thpok gene. In order to replace E4p sequence in the $C d 4$ locus with the two separately located enhancers in the Thpok locus, we synthesized an Eth DNA fragment in which core sequences of TE and $P E$ were conjugated (Supplementary Fig. 1a), and generated a $C d 4^{\text {Eth }}$ allele through homologous recombination in embryonic stem (ES) cells (Fig. 1a and Supplementary Fig. 1b).

CD4 expression on $C d 4^{\text {Eth/Eth }}$ thymocytes at the DP stage, defined as the CD24 ${ }^{\text {hi }}$ TCR $\beta^{\text {lo }}$ population, was lower than that in control $C d 4^{+/+}$but higher than that in $C d 4^{\Delta E 4 p / \Delta E 4 p}$ cells (Fig. 1b, c). Given that the activity of TE and $P E$ in the Thpok locus was low in pre-selection DP thymocytes and gradually increased during thymocytes maturation ${ }^{13,14,16}$, Eth activity in the $C d 4$ locus was likely to retain original stage-specificity and not be sufficient to completely restore CD4 expression in precursor DP thymocytes. However, during maturation into the helperlineage, the CD4 expression level from the $C d 4^{\text {Eth }}$ allele closely approached that from the wild-type Cd4 allele (Fig. 1b, c, and Supplementary Fig. 1c).

We next examined whether CD4 expression from the Cd4 $4^{E t h}$ allele could be stably maintained after cell division. Although the CD4 expression level from the $C d 4^{\triangle E} 4 p$ allele was decreased according to the rounds of cell divisions as previously reported $^{11,12}, \mathrm{CD} 4$ expression was retained at a higher level in $\mathrm{CD} 4^{+}$cells from $C d 4^{E t h / E t h}$ mice (Fig. 1d). DNA de-methylation at the intronic region was induced in $C d 4^{E t h / E t h}$ cells to a similar extent as that in control $C d 4^{+/+}$cells, whereas DNA remained significantly methylated in $C d 4^{\Delta E 4 p / \Delta E 4 p}$ cells (Fig. 1e). These observations indicated that the synthetic heterologous Eth enhancer was able to restore E4p function in the Cd4 locus to a degree sufficient to establish a heritable actives state at least in part through the induction of DNA de-methylation.

Characterization of the $\boldsymbol{C d} 4$ maturation enhancer $(\mathbf{E 4 m})$. In the murine $C d 4$ locus, the presence of a $C d 4$ maturation enhancer $(E 4 m)$ shortly downstream of $S 4$ has been predicted ${ }^{17}$. We found conserved sequences at approximately $1 \mathrm{~kb}$ downstream of the $S 4$ region (Supplementary Fig. 2a); moreover, the public ATAC-Seq database showed that this region become more accessible specifically in CD4-lineage cells after positive selection, whereas the $S 4$ region became more accessible in CD8-lineage cells (Supplementary Fig. 2a). In addition, our chromatin immunoprecipitation (ChIP) combined with sequencing (ChIP-Seq) analysis detected binding of Runx complexes to $S 4$ and its downstream region in total thymocytes (Fig. 2a). Furthermore, changes of post-translational modifications on $\mathrm{K} 27$ of histone 3 for trimethylation (H3K27me3) and for acetylation (H3K27ac), which are known as markers for inactive and active enhancer states ${ }^{18}$, respectively, indicated that $\mathrm{H} 3 \mathrm{~K} 27 \mathrm{ac}$ at $E 4 p$ was highest in precursor DP thymocytes, whereas $\mathrm{H} 3 \mathrm{~K} 27 \mathrm{ac}$ was increased at a the putative $E 4 m$ region in post-selection $\left(\mathrm{CD} 69^{+} \mathrm{CD} 24^{\text {hi }} \mathrm{TCR}^{+}\right)$ thymocytes. In comparison, $\mathrm{H} 3 \mathrm{~K} 27 \mathrm{me} 3$ modification accumulated at both regions specifically in $\mathrm{CD} 8^{+} \mathrm{T}$ cells. Together, these features prompted us to test the function of this putative $E 4 \mathrm{~m}$ region genetically by deleting the $337 \mathrm{bp}$ core conserved sequences. Accordingly, by using an ES cell clone harboring a $C d 4^{+/+}$or $C d^{\Delta S 4 / \Delta E 4 p}$ genotype (Supplementary Fig. 2b, c), we established two mouse lines harboring $C d 4^{\Delta E 4 m}$ and $C d 4^{\Delta E 4 p \text { : }}$ $\triangle E 4 m$ alleles, with the latter lacking both $E 4 p$ and $E 4 m$ 
a

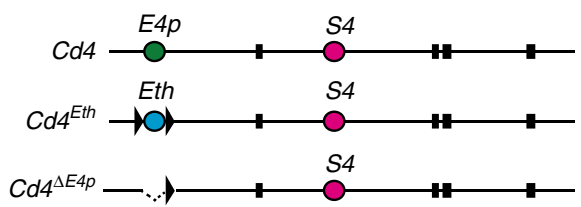

b

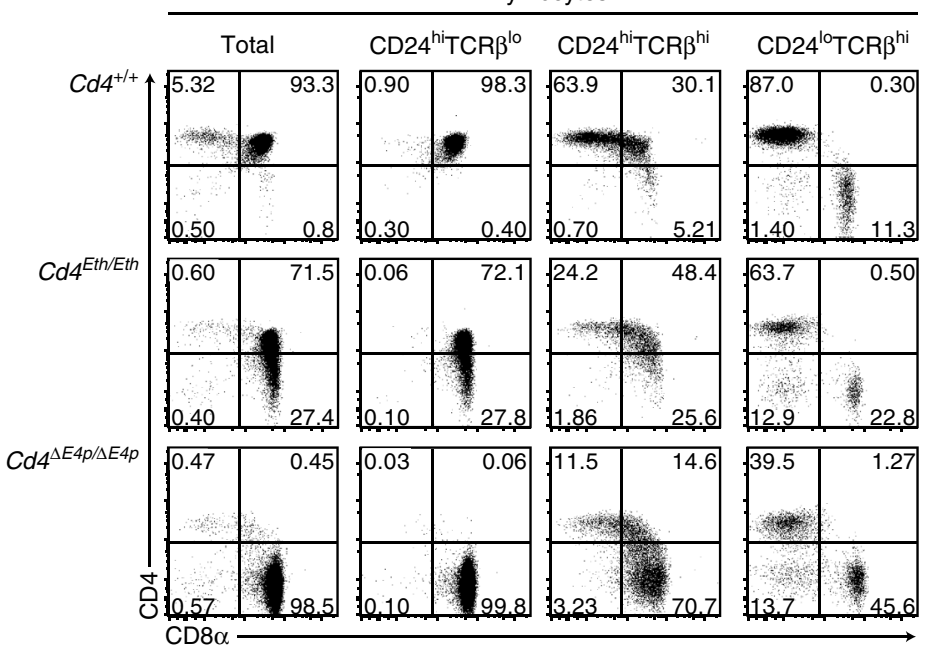

C

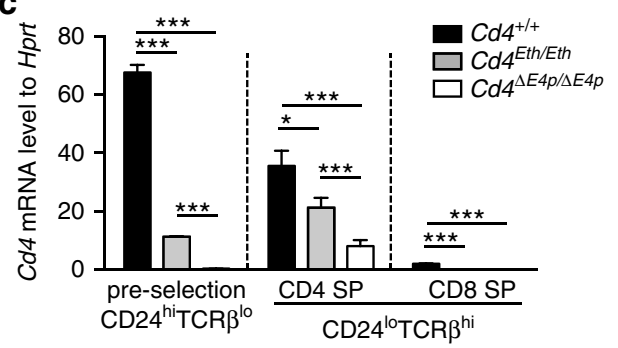

d

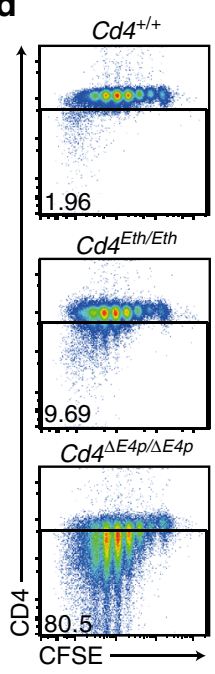

e

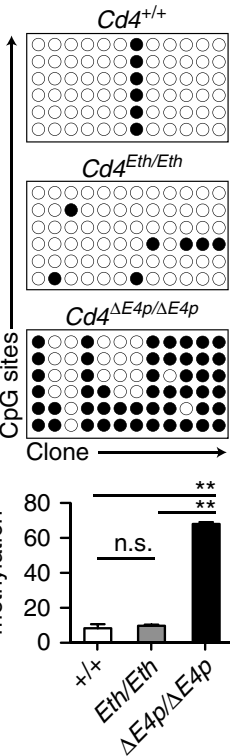

Fig. 1 Enhancer replacement between $C d 4$ and Thpok genes. a Schematic structures of mutant $C d 4$ alleles. Ovals marked with different colors represent cisregulatory regions, $C d 4$ silencer $(S 4), C d 4$ proximal enhancer $(E 4 p)$, and synthetic Thpok enhancer (Eth). The black box and triangle represent exons and loxP sequences, respectively. b Dot plots showing CD4 and CD8 expression and histograms at the right showing CD4 expression in indicated cell subsets from mice with indicated genotypes. Numbers in quadrants indicate respective cell percentage. Representative results of at least three independent analyses. c Graph showing relative Cd4 to Hprt mRNA in pre-selection CD24hiTCR $\beta^{\text {lo }}$ thymocytes, CD24loTCR $\beta^{\text {hi }}$ CD4 single positive (SP), and CD24 ${ }^{\text {loTCR }} \beta^{\text {hi }}$ CD8 SP thymocytes of mice with indicated genotypes. Means \pm SD. ${ }^{\star \star \star} p<0.001,{ }^{\star} p<0.05$ ((One-way ANOVA and Tukey's multiple comparison test)). d Pseudocolor plots showing CD4 expression and cell divisions five days after in vitro stimulation of sorted CD4 ${ }^{+} \mathrm{T}$ cells from mice with indicated genotype. Numbers in the indicated gate represent cell percentages. e Bisulfite PCR at six amplicons from the first intron in the $C d 4$ gene in naïve $\mathrm{CD}^{+} \mathrm{T}$ cells from mice with indicated genotypes. Symbols indicate methylated (black filled circle) or un-methylated (black open circle) $\mathrm{CpG}$ motifs. The lower graph shows the summary of three independent experiments. Means $\pm \mathrm{SD}$. ${ }^{\star \star \star} p<0.01$ (unpaired student $t$ test, two-sided)

(Supplementary Fig. 2b, c). Strikingly, CD4 expression during T cell development was completely abolished in the $C d 4^{\Delta E 4 p: \Delta E 4 m /}$ $\triangle E 4 p: \triangle E 4 m$ mice (Fig. 2b). This finding demonstrated not only that enhancer activity complementing CD4 expression $(E 4 m)$ from the $C d 4^{\Delta E 4 p}$ allele is embedded on the deleted region, but also that $E 4 p$ and E4m serve as major enhancers to drive CD4 expression in $\mathrm{T}$ cells.

We next examined the impact of the $E 4 m$ deletion alone on CD4 expression and T cell development in $C d 4^{\Delta E 4 m / \Delta E 4 m}$ mice. Although the CD4 expression level at the DP stage was compatible between $C d 4^{+/+}$and $C d 4^{\Delta E 4 m / \Delta E 4 m}$ cells, it became lower in $C d 4^{\Delta E 4 m / \Delta E 4 m}$ cells than that in $C d 4^{+/+}$cells at the CD8 ${ }^{-} \mathrm{CD} 24^{\text {hi }}$ TCR $\beta^{\text {hi }}$ stage (Fig. 2b, c). Notably, CD4 expression level from the $C d 4^{\Delta E 4 p}$ allele was higher than that from the $C d 4^{\Delta E 4 m}$ allele in $\mathrm{CD} 8^{-} \mathrm{CD} 24^{\mathrm{hi}} \mathrm{TCR} \beta^{\text {hi }}$ cells, although CD4 expression levels from these two mutant $C d 4$ alleles became similar to each other and lower than that from the wild-type $C d 4$ allele in mature $\left(\mathrm{CD} 24^{\mathrm{lo}} \mathrm{TCR} \beta^{\mathrm{hi}}\right) \mathrm{CD} 8^{-}$thymocytes. Thus, the $C d 4^{\Delta E 4 p}$ allele could induce CD4 expression faster than the Cd4 ${ }^{\Delta E 4 m}$ allele after positive selection, although changes of histone modification patterns at the E4p was equivalent between control $C d 4$ and $C d 4^{\Delta E 4 m}$ alleles (Supplementary Fig. 3).
Following activation of $\mathrm{CD}^{+} \mathrm{CD}^{-}$splenic $\mathrm{T}$ cells, CD4 expression from the $C d 4^{\Delta E 4 m}$ allele decreased to a similar extent as that from the $C d 4^{\Delta L S}$ allele (Fig. 2d), in which both $S 4$ and $E 4 m$ were deleted ${ }^{6}$. However, the percentage of cells retaining CD4 expression was higher in $C d 4^{\Delta E 4 m / \Delta E 4 m}$ cells than in $C d 4^{\Delta E 4 p / \Delta E 4 p}$ cells, although the percentage of methylated CpG motifs at intronic regions remained higher in these two cell types to a similar extent (Figs $1 \mathrm{c}$ and 2e). These results indicated that $E 4 \mathrm{~m}$ activity is also necessary to establish a heritable active state at the $C d 4$ locus in part by activating the DNA de-methylation process together with E4p.

Of note, a percentage of $\mathrm{CD}^{+}{ }^{+} \mathrm{CD} 8^{-}$cells among the mature thymocyte and lymph node $\mathrm{T}$ cell population was decreased in $C d 4^{\Delta E 4 m / \triangle E 4 m}$ mice (Fig. 2b, f). To examine whether re-directed differentiation of MHC-II selected cells was involved in the decrease of $\mathrm{CD}^{+} \mathrm{T}$ cells, as was observed in $C d 4^{\Delta E 4 p / \Delta E 4 p}$ mice $^{11}$, we analyzed the differentiation of MHC-II selected cells under $\beta 2$-microglobulin-deficient condition. In CD24 $4^{\mathrm{lo}} \mathrm{TCR} \beta^{\text {hi }}$ thymocyte population, while $\mathrm{CD} 4^{-} \mathrm{CD} 8^{+}$cells were undetectable

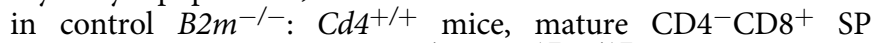
thymocytes emerged in $B 2 m^{-/-}: C d 4^{\Delta E 4 m / \Delta E 4 m}$ mice (Fig. 3a, b). In the CD24 ${ }^{\text {hi }} \mathrm{TCRb}^{\text {hi }}$ population, the percentages of CD8 
a
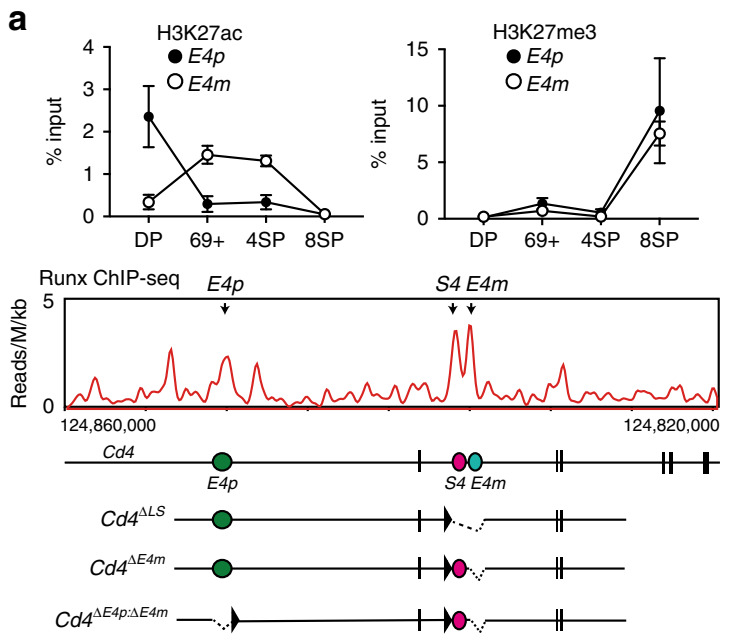

C

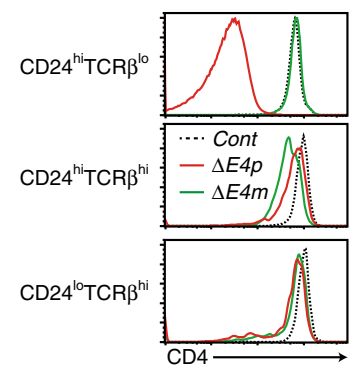

d

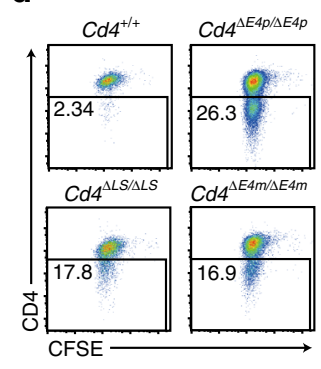

b

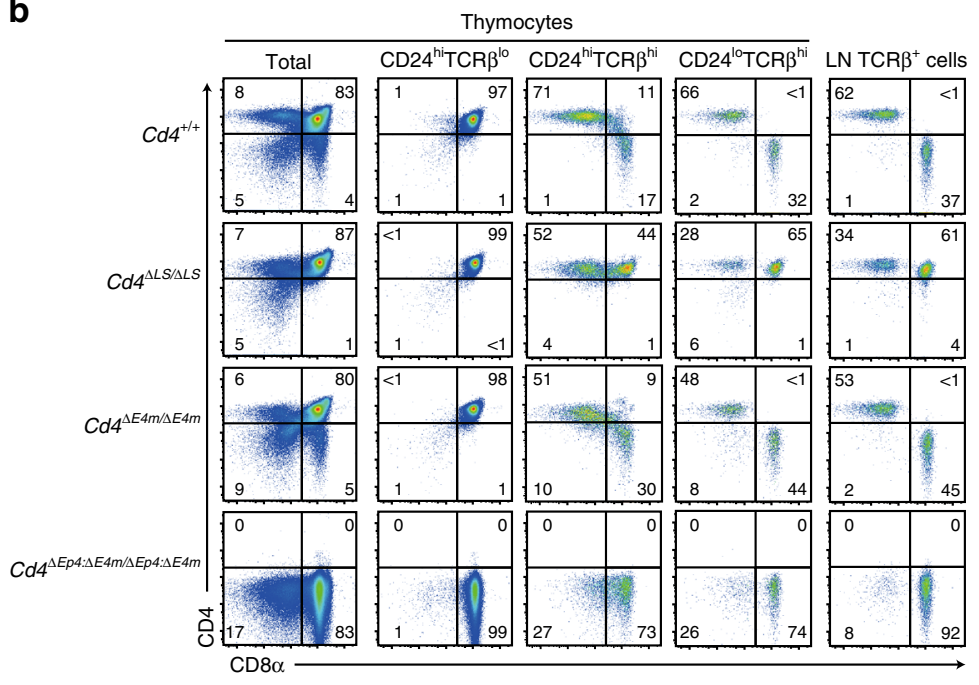

e

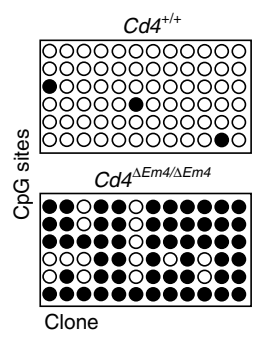

f

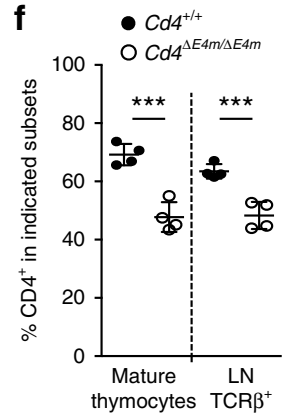

Fig. 2 Effects of loss of the maturation enhancer, E4m, on CD4 expression. a Upper graphs showing kinetic changes of H3K27ac and H3K27me3 at E4p and E4m regions in pre-selection (DP), freshly selected (CD69+), and CD4 SP (4SP) and CD8 SP (8SP) thymocytes. Summary of three independent ChIP experiments. Means \pm SD. (Middle) Runx ChIP-Seq track at the Cd4 gene in total thymocytes. Gene structures and regulatory regions are shown as in Fig. 1a. Schematic structures of mutant Cd4 alleles are shown at the bottom. b Pseudocolor plots showing CD4 and CD8 expression in indicated cell subsets from mice with indicated genotypes. Numbers in quadrants indicate respective cell percentages. Representative results of at least three independent analyses of each genotype. $\mathbf{c}$ Histograms showing CD4 expression levels on CD24 ${ }^{\text {hiTCR }} \beta^{\text {lo }}$, CD8-negative CD24hiTCR $\beta^{\text {hi }}$ and CD8-negative

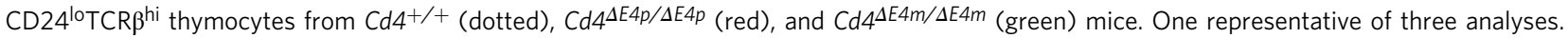

d Pseudocolor plots showing CD4 expression and cell divisions five days after in vitro stimulation of sorted peripheral CD4 ${ }^{+} \mathrm{T}$ cells from mice with indicated genotypes. Numbers in the indicated gate represent cell percentages. e Bisulfite PCR analyses of naïve CD4 ${ }^{+} \mathrm{T}$ cells from Cd4 ${ }^{+} /+$and $C d 4^{4 E 4 m / ~}$ $\Delta E 4 \mathrm{~m}$ mice are shown as in Fig. 1d. The right graph shows a summary of three independent experiments. Means \pm SD. ${ }^{\star \star} p<0.01$ (unpaired student $t$ test, two-sided). $\mathbf{f}$ Graph showing percentage of $\mathrm{CD} 4^{+} \mathrm{CD} 8^{-}$cells in mature (CD24 $\left.{ }^{\mathrm{lo}} \mathrm{TCR} \beta^{\text {hi }}\right)$ thymocytes and the lymph node TCR $\beta^{+}$population of $\mathrm{Cd} 4{ }^{+/+}$and $C d 4^{\Delta E 4 m / \Delta E 4 m}$ mice. Means $\pm \mathrm{SD}$. ${ }^{\star *} p<0.001$ (unpaired student $t$ test, two-sided)

expressing cells were also higher in $B 2 \mathrm{~m}^{-/-}: C d 4^{\Delta E 4 m / \Delta E 4 m}$ mice (Fig. 3a). As ThPOK is involved in terminating CD8 expression during the maturation of MHC-II selected cells ${ }^{19,20}$, we analyzed Thpok expression using the Thpokgfp reporter allele ${ }^{16}$. Although the majority of MHC-II selected CD24hiTCRb ${ }^{\text {hi }}$ thymocytes in $B 2 m^{-1-}: C^{+/+}$mice expressed Thpok-gfp at a higher level, biphasic and lower Thpok-gfp expression was observed in those cells in $B 2 m^{-1-}: C d 4^{\Delta E 4 m} / \Delta E 4 m$ mice (Fig. 3c), and the CD8 expression level remained higher in Thpok-gfp lo cells than in Thpok-gfp ${ }^{\text {hi }}$ cells (Fig. 3c). These observations suggested that a low and delayed CD4 expression after positive selection in the absence of $E 4 m$ resulted in a re-direction of some MHC-II selected thymocytes to the CD8-lineage in part through impaired Thpok induction.

However, the percentage of $\mathrm{CD} 4^{-} \mathrm{CD}^{+}$subset cells declined in the peripheral lymphoid tissues of $B 2 m^{-1-}: C d 4^{\Delta E 4 m / \Delta E 4 m}$ mice (Fig. 3b). This might be explained by the differentiation of CD4 ${ }^{-} \mathrm{CD} 8{ }^{+}$thymocytes into $\mathrm{CD} 4{ }^{+} \mathrm{CD} 8{ }^{-} \mathrm{T}$ cells or a defect in the survival of $\mathrm{CD}^{-} \mathrm{CD} 8^{+}$thymocytes in those mice. To trace CD4 and $\mathrm{CD} 8$ expression, we cultured $\mathrm{CD}^{-} \mathrm{CD}^{+}$thymocytes prepared from $B 2 m^{-1-}: C d 4^{\Delta E 4 m / \Delta E 4 m}$ mice and found that these cells remained as $\mathrm{CD}^{-} \mathrm{CD}^{+}$cells after five days (Fig. $3 \mathrm{~d}$ ). It has been shown that IL-7 signaling is crucial for the survival and expansion of CD8 SP thymocytes ${ }^{21,22}$. Consistent with a lower IL7R expression level in the $\mathrm{CD} 4^{-} \mathrm{CD} 8^{+}$thymocytes of $B 2 \mathrm{~m}^{-/-}$: $C d 4^{\Delta E 4 m / \Delta E 4 m}$ mice than in control MHC-I restricted CD4 ${ }^{-} \mathrm{CD} 8^{+}$ thymocytes (Fig. 3e), the phosphorylation of Stat5 after in vitro IL7 stimulation was reduced in $B 2 m^{-/-}: C d 4^{\Delta E 4 m / \triangle E 4 m} \mathrm{CD} 4^{-} \mathrm{CD} 8^{+}$ thymocytes (Fig. 3e). These results suggested that the low level of IL-7R expression in re-directed $\mathrm{CD} 4^{-} \mathrm{CD} 8^{+}$thymocytes is involved in the decline in the percentage of $\mathrm{CD} 4^{-} \mathrm{CD} 8^{+}$subset in the periphery of $B 2 m^{-/-}: C d 4^{\Delta E 4 m / \Delta E 4 m}$ mice.

Bcl11b is essential for activation of E4p and E4m enhancers. To understand how $E 4 m$ function is regulated, it is necessary to identify molecules that bind and regulate $E 4 m$ activity. During analyses of Bcl11b function ${ }^{23}$, we observed that Bcl11b associates with the E4p, S4, and E4m regions in total thymocytes (Fig. 4a). ChIP-qPCR showed that associations of both Runx and Bcl11b with the E4p were dramatically decreased in peripheral $\mathrm{T}$ cells (Fig. 4b). Runx binding to $S 4$ was the highest in $\mathrm{CD}^{+} \mathrm{T}$ cells but 

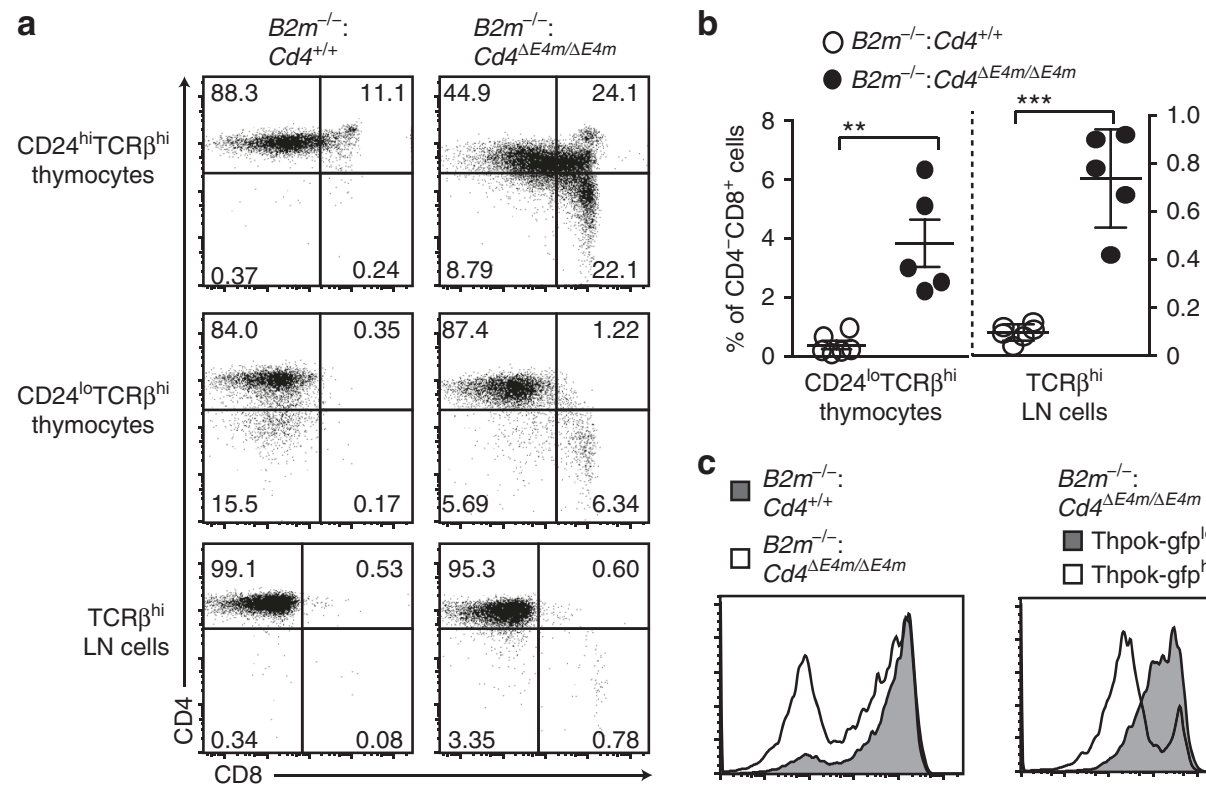

Thpok-gfp
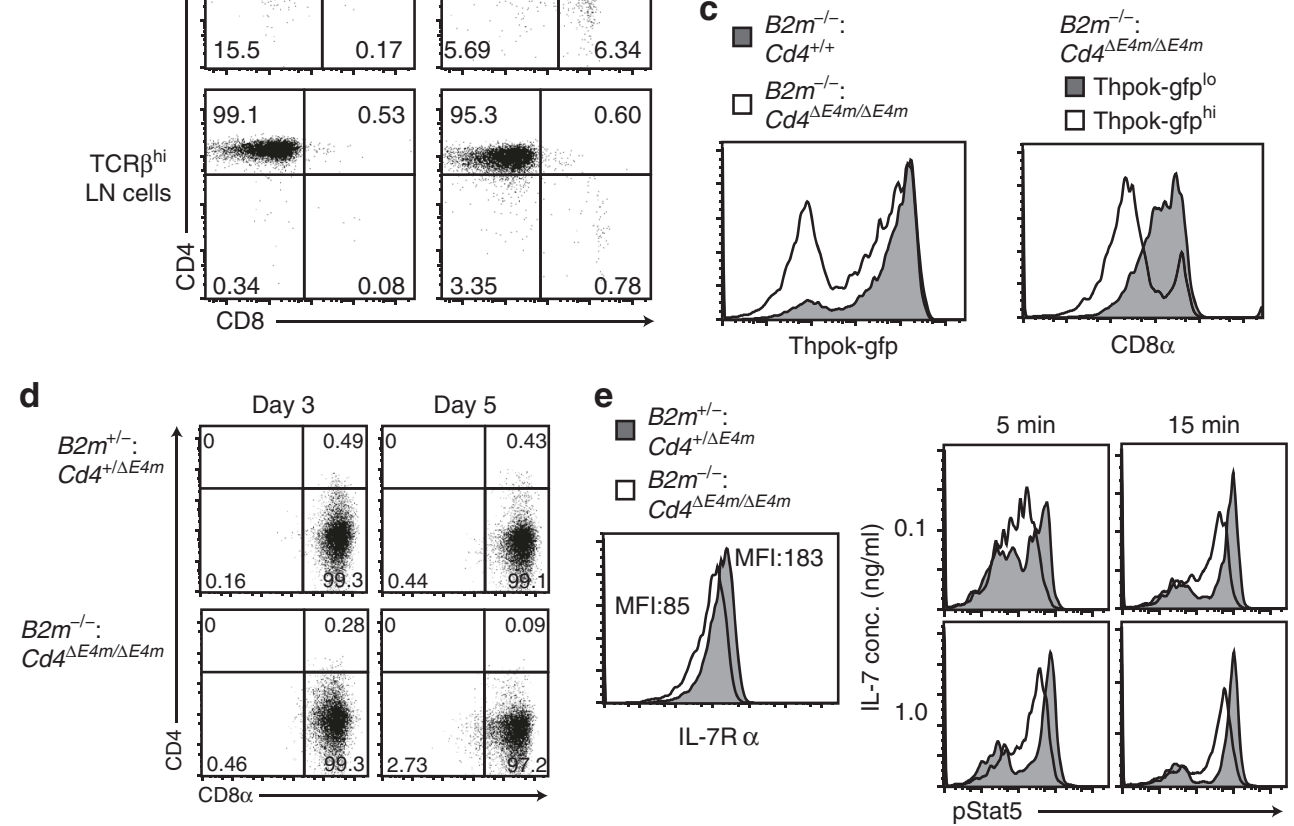

Fig. 3 Re-directed differentiation of MHC-II selected thymocyte by loss of the E4m enhancer. $\mathbf{a}$, $\mathbf{b}$ Dot plots in a showing CD4 and CD8 expression in indicated cell subsets from $B 2 \mathrm{~m}^{-/-}: \mathrm{Cd} 4^{+/+}$and $B 2 \mathrm{~m}^{-/-}: \mathrm{Cd} 4^{\Delta E 4 \mathrm{~m} / \Delta E 4 \mathrm{~m}}$ mice. Graphs in $\mathbf{b}$ showing summary of percentage of CD4-CD8 ${ }^{+}$cells in mature (CD24 ${ }^{\text {lo } T C R ~} \beta^{\text {hi }}$ ) thymocytes and the lymph node (LN) TCR $\beta^{+}$population. Means \pm SD. ${ }^{\star \star} p<0.01,{ }^{\star \star \star} p<0.001$ (unpaired student $t$ test, two-sided). c Histogram at left showing Thpok-gfp expression in freshly-selected (CD24hiTCR $\beta^{\text {hi }}$ ) thymocytes of $B 2 \mathrm{~m}^{-/-}: \mathrm{Cd} 4^{+/+}$and $B 2 \mathrm{~m}^{-/-}: \mathrm{Cd} 4^{4 E 4 m / \Delta E 4 m}$ mice. Histogram at right showing CD8 expression in Thpok-gfplo and Thpok-gfp ${ }^{\text {hi }} \mathrm{CD} 24^{\text {hiTCR }}{ }^{\text {hi }}$ thymocytes of $B 2 \mathrm{~m}^{-/-}: \mathrm{Cd} 4^{\Delta E 4 \mathrm{~m} / \Delta E 4 \mathrm{~m}}$ mice. One representative result of two mice. $\mathbf{d}$ Dot plots showing CD4 and CD8 expression three and five days after TCR stimulation of sorted $C D 4^{-} \mathrm{CD} 8{ }^{+}$mature thymocytes from $B 2 \mathrm{~m}^{+/-}: \mathrm{Cd} 4^{+/ \Delta E 4 m}$ and $B 2 \mathrm{~m}^{-/-}: \mathrm{Cd} 4^{\Delta E 4 \mathrm{~m} / \Delta E 4 \mathrm{~m}}$ mice. Numbers in quadrants indicate respective cell percentages. e IL7R expression in CD4 ${ }^{-} \mathrm{CD} 8^{+}$ thymocytes (left) and phosphorylated Stat5 levels in CD4 ${ }^{-} \mathrm{CD} 8^{+}$thymocytes (right) at 5 and 15 min following IL7 stimulation at indicated concentrations from $B 2 \mathrm{~m}^{+/-}: \mathrm{Cd} 4^{+/ \Delta E 4 m}$ and $B 2 \mathrm{~m}^{-/-}: \mathrm{Cd} 4 \Delta E 4 \mathrm{~m} / \Delta E 4 \mathrm{~m}$ mice are shown. Numbers in left histograms indicate mean fluorescence intensity (MFI)

was almost lost in $\mathrm{CD}^{+} \mathrm{T}$ cells, with Bcl11b binding to $S 4$ also becoming lower in $\mathrm{CD} 4^{+} \mathrm{T}$ cells compared to that in other cell subsets (Fig. 4b). Although the association of Runx to E4m was compatible between the three cell subsets, Bcl11b binding to $E 4 m$ was higher in $\mathrm{CD}^{+} \mathrm{T}$ cells than in $\mathrm{CD}^{+} \mathrm{T}$ cells (Fig. $4 \mathrm{~b}$ ).

Considering such Bcl11b associations with $C d 4$ regulatory regions, we examined $\mathrm{Bcl} 1 \mathrm{lb}$ function in regulating $\mathrm{Cd} 4$ expression by using two $B c l 11 b$ mutant models. We recently generated a hypomorphic $B c l 11 b$ allele, referred to as $B c l 11 b^{m}$, that produces truncated Bcl11b protein lacking the last zincfinger domain ${ }^{23}$. In thymi of newborn $B c l 11 b^{m / m}$ homozygous mice, which die at two days after birth, $\mathrm{CD} 4{ }^{\text {lo }} \mathrm{CD} 8{ }^{+}$cells were present in the $\mathrm{CD} 24^{\text {hi }} \mathrm{TCRb}^{\text {lo }}$ population, whereas nearly all $\mathrm{CD} 24^{\text {hi }} \mathrm{TCRb}^{\text {lo }}$ thymocytes appeared as $\mathrm{CD}^{+}{ }^{+} \mathrm{CD} 8{ }^{+} \mathrm{DP}$ cells in control mice (Fig. 4c, d). In addition, CD4 expression in the TCR $\beta^{\text {hi }}{ }^{-} D 8^{-}$mature thymocyte population became lower, resulting in the generation of the $\mathrm{CD}^{-}{ }^{-} \mathrm{CD} 8^{-}$instead of $\mathrm{CD} 4$ ${ }^{+} \mathrm{CD}^{-}$subset in Bcll1 $b^{\mathrm{m} / \mathrm{m}}$ thymi (Fig. $4 \mathrm{c}, \mathrm{d}$ ). We next examined the effect of conditional inactivation of $B c l 11 b$ at DP stage by a Cd4-Cre transgene, and observed emergence of the $\mathrm{CD} 4^{-} \mathrm{CD} 8^{-}$ subset in the CD24 ${ }^{\text {lo }} \mathrm{TCR} \beta^{\text {hi }}$ mature thymocyte population of $B c l 11 b^{F / F}$ : Cd4-Cre mice (Fig. 4e). In order to further address whether Bcl11b regulates $E 4 m$ activity, we then combined E4pdeficiency with Bcll1b inactivation. Under an E4p-deficient background, the CD4 expression level in the mature CD8negative thymocyte population severely decreased upon loss of Bcl11b (Fig. 4e, f). In contrast, CD4 expression level was partially restored after egress from the thymus, with $\mathrm{CD}^{+}{ }^{+} \mathrm{T}$ cells

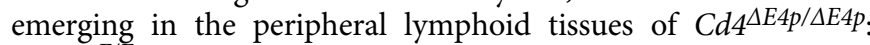
$B c l 11 b^{F / F}$ : Cd4-Cre mice (Fig. $4 \mathrm{e}, \mathrm{f}$ ). However, the CD4 expression in $\mathrm{CD}_{4}{ }^{+} \mathrm{T}$ cells that developed in $B c l 11 b^{F / F}$ : Cd4-Cre mice were unstable after activation-induced cell divisions (Fig. 4g). Thus, dysfunction of Bcl11b resulted in a delayed CD4 induction at two transitional stages, one for becoming DP thymocytes and the other for becoming mature CD4 SP thymocytes, which require activation of E4p and $E 4 m$, respectively.

Characterizing specific activity of $\mathbf{E 4 m}$. In order to further characterize $E 4 m$ activity, we addressed how $E 4 m$ alone behaves 
a

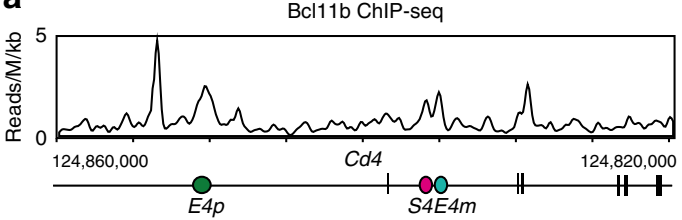

C

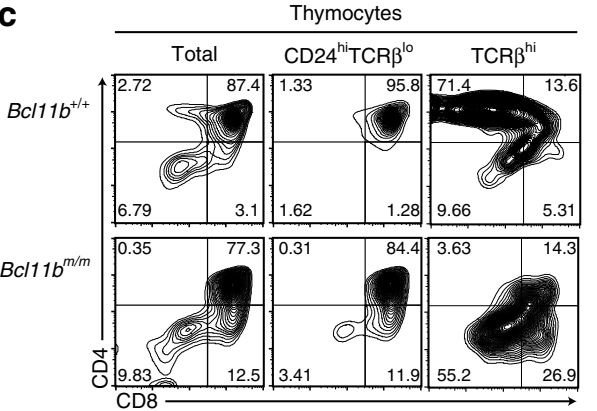

d $\mathrm{OBC} / 11 b^{+/+}$

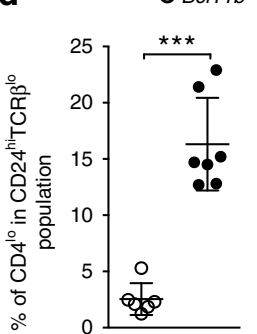

e

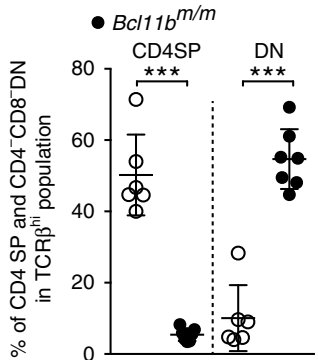

b
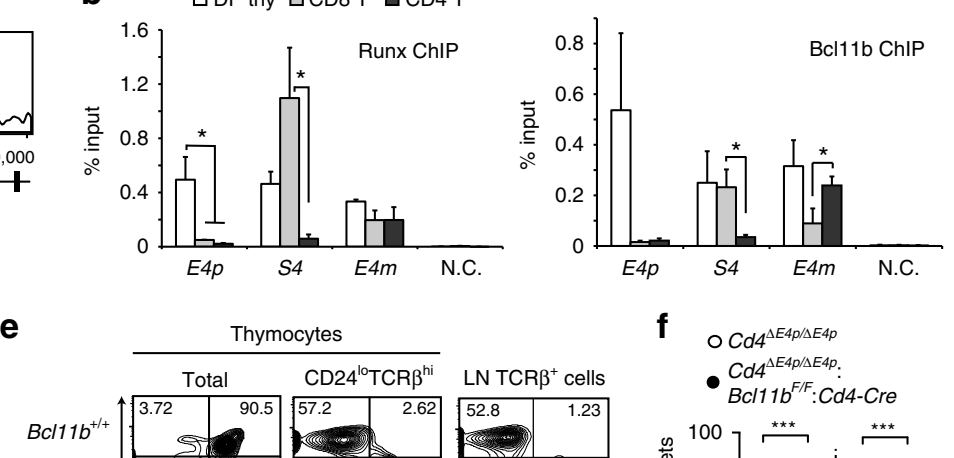

$$
T
$$

Thymocytes
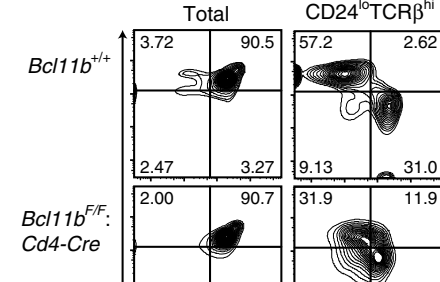

2
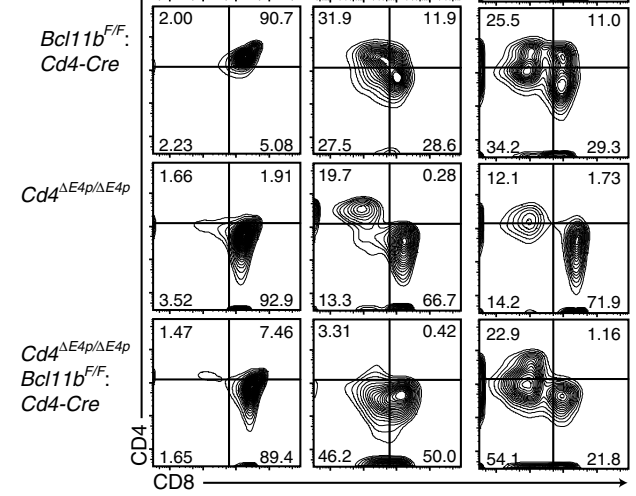

f

f $\bigcirc C d 4^{\triangle E A P \triangle A E 4 P}$

- $C d 4^{\triangle E 4 P \triangle A E 4 P \text { : }}$

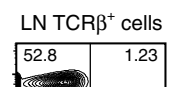

- $B$ Cl11 $b^{F / F}:$ Cd4-Cre
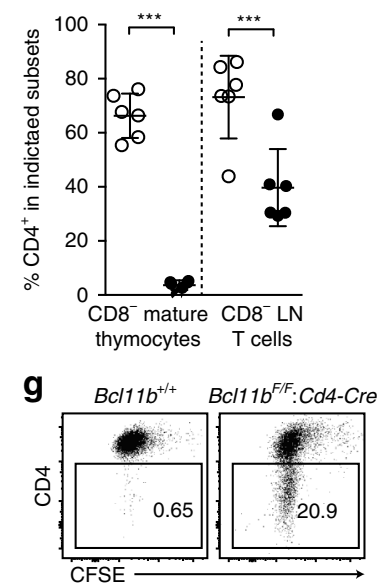

Fig. 4 Requirement for Bcl11b in activating Cd4 at two stages. a ChIP-Seq tracks at the Cd4 gene showing binding of Bcl11b in total thymocytes. b ChIP-qPCR analyses for Runx (left) and Bcl11b (right) bindings to the Cd4 proximal enhancer (E4p), Cd4 silencer (S4), and Cd4 maturation enhancer (E4m) in DP thymocytes, peripheral $\mathrm{CD}^{+}$, and $\mathrm{CD} 8^{+} \mathrm{T}$ cells. N.C.: negative control region. Summary of three independent ChIP experiments. Means $\pm \mathrm{SD}$. ${ }^{\star} p<0.05$ (One-way ANOVA and Tukey's multiple comparison test). c Contour plots showing CD4 and CD8 expression in indicated thymocyte subsets of Bc/17b ${ }^{+/+}$ and $B c / 11 b^{m / m}$ newborn mice. Numbers in quadrants indicate respective cell percentages. Representative results of at least three independent analyses. d Summary of percentage of indicated cell types in indicated cell populations of $B c / 11 b^{+/+}$and $B c 117 b^{m / m}$ mice. Means \pm SD. ${ }^{\star \star \star} p<0.001$ (unpaired student $t$ test, two-sided). e Contour plots showing CD4 and CD8 expression in indicated thymocyte subsets and lymph node (LN) T cells of mice with indicated genotypes. Numbers in quadrants indicate respective cell percentages. Representative results of at least three independent analyses. $\mathbf{f}$ Statistical summary

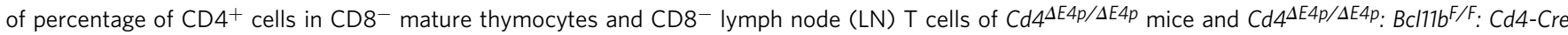
mice mice. Means \pm SD. ${ }^{\star \star \star} p<0.001$ (unpaired student $t$ test, two-sided). $\mathbf{g}$ Dot plots showing CD4 expression and cell divisions five days after in vitro stimulation of sorted $\mathrm{CD}^{+}{ }^{+} \mathrm{T}$ cells from mice with indicated genotypes. One representative of three experiments

by generating a $C d 4^{\Delta E 4 p: \Delta S 4}$ allele, in which only the $E 4 m$ among three regulatory regions remains in the $C d 4$ gene (Fig. 5a). Notably, the percentage of cells expressing CD4 was higher in CD8-negative cells than in CD8-positive cells in the mature thymocyte population of $C d 4 \Delta E 4 p: \Delta S 4 / \Delta E 4 p: \Delta S 4$ mice (Fig. $5 \mathrm{~b}, \mathrm{c}$ ), although it was equally high in both types of cells of $C d 4^{\Delta S 4 / \Delta S 4}$ mice. To exclude a possibility that deleted sequences around $S 4$ in the $C d 4^{\Delta E 4 p: \Delta S 4}$ allele include a functional element for $E 4 m$ activity, we targeted specific mutations onto two Runx-motifs within $S 4$ to abrogate $S 4$ function by a more specific manner ${ }^{9}$, generating a $C d 4^{\Delta E} 4 p: S 4 M$ allele (Fig. 5a). In $C d 4^{\Delta E} 4 p: S 4 M / \triangle E 4 p: S 4 M$ mice, the percentage of cells expressing CD4 remained higher in $\mathrm{CD}^{-}$cells compared to $\mathrm{CD} 8^{+} \mathrm{T}$ cells (Fig. 5b, c). Such $\mathrm{CD} 8^{+}$ cells were almost undetected in $B 2 m^{-1-}: C d 4^{\Delta E 4 p: S 4 M / \triangle E 4 p: S 4 M}$ mice (Supplementary Fig. 4), indicating that the majority of $\mathrm{CD}^{+}$cells in $C d 4^{\Delta E 4 p: S 4 M / \Delta E 4 p: S 4 M}$ mice constituted MHC-I restricted cells. Given that CD4 expression from the $C d 4^{\Delta E 4 p: \Delta S 4}$ allele is likely to depend on $E 4 m$, these observations indicate that the $E 4 m$ activity is more predominant in the helper-lineage cells than in cytotoxic-lineage cells.

Regulation of $\mathbf{E 4 m}$ activity by Runx complexes. To examine how such helper-lineage dominant activity of $E 4 m$ is regulated, we analyzed the function of transcription factors that are known to be involved in lineage-specific expression of the $C d 4$ gene. Two Runx proteins, Runx1 and Runx3, are essential to control lineage specific $C d 4$ expression through activating the $S 4$ silencer ${ }^{9}$. The penta-peptide sequences, VWRPY, at the C-terminal end of Runx proteins serve as a platform to recruit the TLE/Groucho corepressor family 24,25 , and are essential for Runx-mediated Cd4 repression ${ }^{10,26}$. Accordingly, mice lacking the VWRPY motif from both Runx1 and Runx3 proteins (Runx1/Runx $3^{\Delta V / \Delta V}$ mice), exhibited full CD4 de-repression in $\mathrm{CD}^{+} \mathrm{T}$ cells, phenocopying the $C d 4^{\Delta S 4 / \Delta S 4}$ mice (Figs $5 \mathrm{~b}$ and $6 \mathrm{a}$ ). Given a loss of $S 4$ activity by Runx1/Runx $3^{\Delta V / \Delta V}$ mutation, we assumed that CD4 expression in Runx1/Runx $3^{\Delta V / \Delta V}: C d 4^{\Delta E 4 p / \Delta E 4 p}$ mice was similar to that

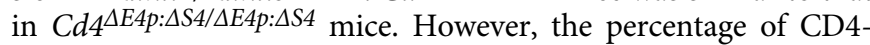
expressing cells was comparable between $\mathrm{CD}^{+}$and $\mathrm{CD} 8^{-}$cells in Runx1/Runx $3^{\Delta V / \Delta V}$ : $C d 4^{\Delta E 4 p / \Delta E 4 p}$ mice (Fig. 6a, b). Thus, a discrepancy existed between the effects from loss of cis-acting $S 4$ and loss of trans-acting Runx proteins on the $C d 4^{\Delta E} 4 p$ allele, suggesting a possible involvement of S4-indpendent mechanisms through which Runx proteins suppress E4m activity. We then generated Runx1/Runx $3^{\Delta V / \Delta V}: C d 4^{\Delta E 4 p: S 4 M / \Delta E 4 p: S 4 M}$ mice and observed an increase of the CD4 expressing population in $\mathrm{CD}^{+}$ T cells (Fig. 6a, b). Our ChIP-qPCR analyses showed that Runx 
a
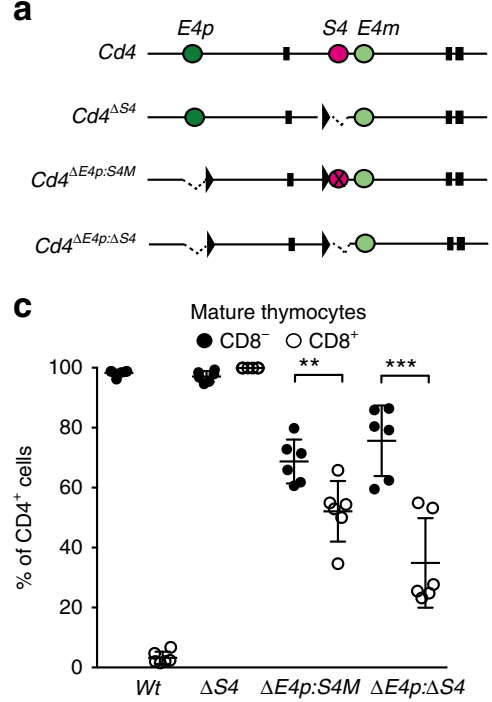

b

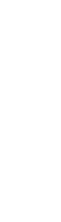

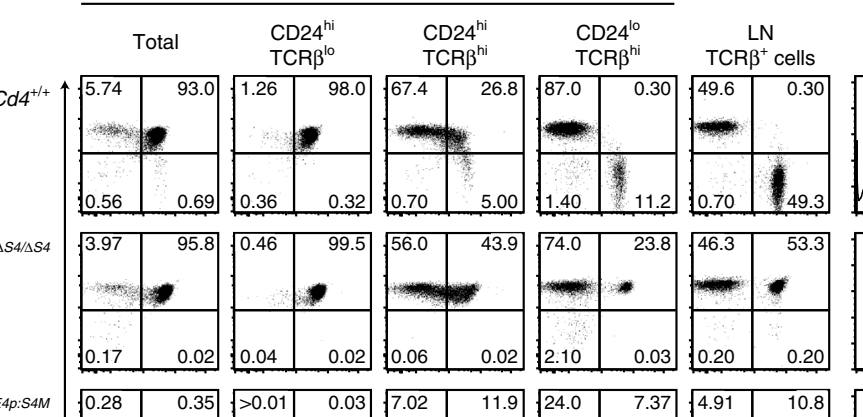

$C d 4^{\Delta E 4 p: S 4 M / \triangle E 4 p: S 4 M}$
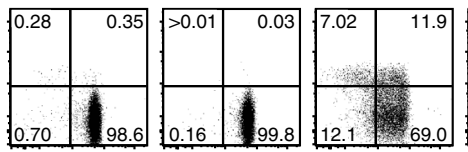

$C d 4^{\Delta E 4 p: \Delta S 4 / \Delta E 4 p: \Delta S 4}$
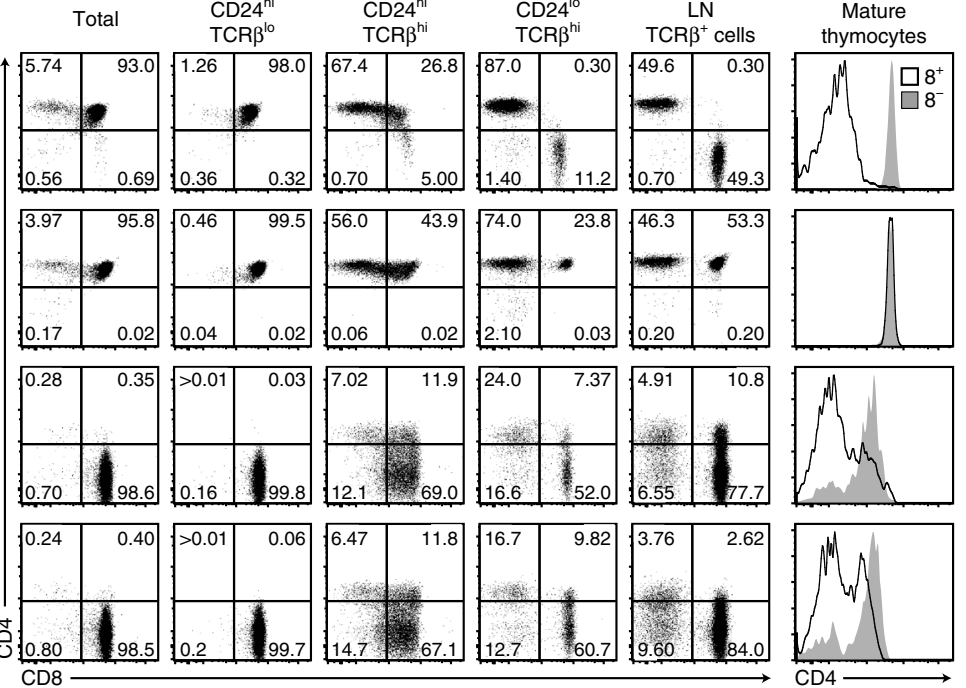

Fig. 5 Helper-lineage dominant activity of E4m. a Schematic structures of Cd4, Cd4 ${ }^{\Delta S 4}$, Cd4 $4 E 4 p: S 4 M$, and Cd4 ${ }^{\Delta E 4 p: \Delta S 4}$ alleles are shown as in Fig. 2 a. Mutations at two Runx sites within S4 are marked as X in the Cd4 4 E4p:S4M allele. b Dot plots showing CD4 and CD8 expression in indicated thymocyte subsets of mice with indicated genotypes. Numbers in quadrants indicate respective cell percentages. Right histograms showing CD4 expression in CD ${ }^{+}$ and $C D 8^{-}$mature (CD24 ${ }^{\text {lo TCR }} \beta^{\text {hi }}$ ) thymocyte populations. Representative results of at least five independent analyses. c Summary of percentage of CD4+

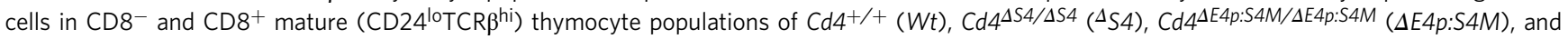

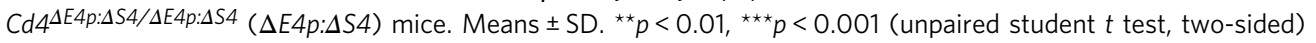

association with the $E 4 m$ was unaffected by eliminating Runxmotifs in S4, whereas Runx binding to $S 4$ was abrogated (Fig. 6c). Together, these observations indicated that Runx proteins are likely to repress $E 4 m$ activity in a VWRPY-dependent manner through their S4-independent association with $E 4 m$.

Lack of canonical Runx-motifs within E4m suggests that recruitment of Runx complexes to $E 4 m$ might be mediated via protein-protein interaction. As both Bcl11b and Satb1 are functional activators for the $E 4 m$ enhancer and interact with Runx proteins ${ }^{23,27}$, we wished to test whether Runx association with $E 4 m$ is affected by lack of Bcl11b and Satb1. However, the severe reduction of mature thymocytes in $B c l 11 b^{F / F}$ : Satb1 $1^{F / F}$ : Cd4-Cre mice (Supplementary Fig. 5a) made it possible to perform ChIP-qPCR analyses only in DP thymocytes. In this setting, Runx binding to $S 4, E 4 p$, and $E 4 m$ was not significantly changed by the loss of Bcl11b and Satblproteins (Supplementary Fig. 5b), suggesting the presence of another factor(s) that recruits Runx complexes to $\mathrm{E} 4 \mathrm{~m}$.

Repressive regulation of $\mathbf{E 4 m}$ by ThPOK. As ThPOK was shown to be essential for the regulation of lineage-specific CD4 expression by counteracting against $S 4$-mediated $C d 4$ repression ${ }^{16,28}$, we next examined whether ThPOK is involved in the regulation

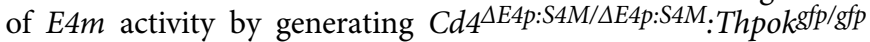
mice that lack ThPOK expression. Notably, loss of ThPOK resulted in an increase of CD4 expressing cells in mature $\mathrm{CD} 8^{+}$ thymocyte and peripheral $\mathrm{CD}^{+} \mathrm{T}$ cell populations (Fig. 7a, b). Increase of the $\mathrm{CD}^{+}{ }^{+}$subset by lack of ThPOK had already been observed at the CD24 ${ }^{\text {hi TCR }} \beta^{\text {hi }}$ stage composed of freshly selected thymocytes and in Thpok-gfp ${ }^{-}$cells that represent MHC-Isignaled ThPOK non-expressing cells. These genetic results suggested that ThPOK restrains $E 4 m$ activity from the early initiation phase. Similar early induction of CD4 in CD24hiTCR $\beta^{\text {hi }}$ thymocytes from the $C d 4^{\Delta E 4 p: S 4 M}$ allele was also observed by Runx1/ Run $\times 3^{\Delta V / \Delta V}$ mutation (Fig. 7c), suggesting that the mechanism by which Runx represses $E 4 m$ activity became non-functional through the lack of ThPOK expression. Our ChIP-qPCR using peripheral $\mathrm{CD}^{+} \mathrm{T}$ cells showed that ThPOK associated with $E 4 m$ in the absence of the $S 4$ (Supplementary Fig. 5c), and Runx association with $\mathrm{E} 4 \mathrm{~m}$ in pre-selection DP thymocytes was not affected by loss of ThPOK (Fig. 7d). Interestingly, level of $\mathrm{H} 3 \mathrm{~K} 27 \mathrm{ac}$ at $E 4 m$ region tended to be higher in $\mathrm{CD}^{ \pm}$mature thymocytes in the absence of ThPOK, although it was lower than that in control CD4 SP thymocytes (Supplementary Fig. 5d). These observations unraveled an unexpected ThPOK function that assists Runx-mediated repression of $E 4 m$ activity after recruitment of Runx to the $E 4 m$ enhancer.

\section{Discussion}

By generating several mutant $C d 4$ alleles, our study provides novel insights into how two enhancers, E4p and $E 4 m$, regulate $C d 4$ expression (Supplementary Fig. 6). E4p and E4m cooperate to establish a stably heritable active state in the helper-lineage $\mathrm{T}$ cells by activating the DNA de-methylation process. It is unclear whether such property is endowed to specific enhancers or is a general feature of enhancers. We showed that a synthetic Eth enhancer derived from the Thpok gene could compensate the E4p activity that induces DNA de-methylation. This suggests that specific sequences within the E4p are unlikely to be absolutely essential to control DNA de-methylation at the $C d 4$ locus. Despite their differences in sequences, it is possible that Eth and E4p poise similar epigenetic codes that can be recognized by common machinery that induces DNA de-methylation. Recent work reported accumulation of $5 \mathrm{hmC}$, which is generated by TET family proteins through DNA de-methylation processs $^{29}$, near the Thpok proximal enhancer core region in the CD4 SP thymocytes $^{30}$, suggesting that TET proteins are involved in DNA demethylation for activation of the Thpok gene. Thus, once distinct protein complexes bound on a distinct enhancer marks the target locus with such epigenetic codes, a sequential reaction that induces DNA de-methylation might be carried out. Alternatively, $E 4 p / E 4 m$ enhancers and Eth were bound by same nuclear protein (s) that recruit machinery to induce DNA de-methylation. Bcl11b and Satb1 may represent candidates for such shared factors, as 
a
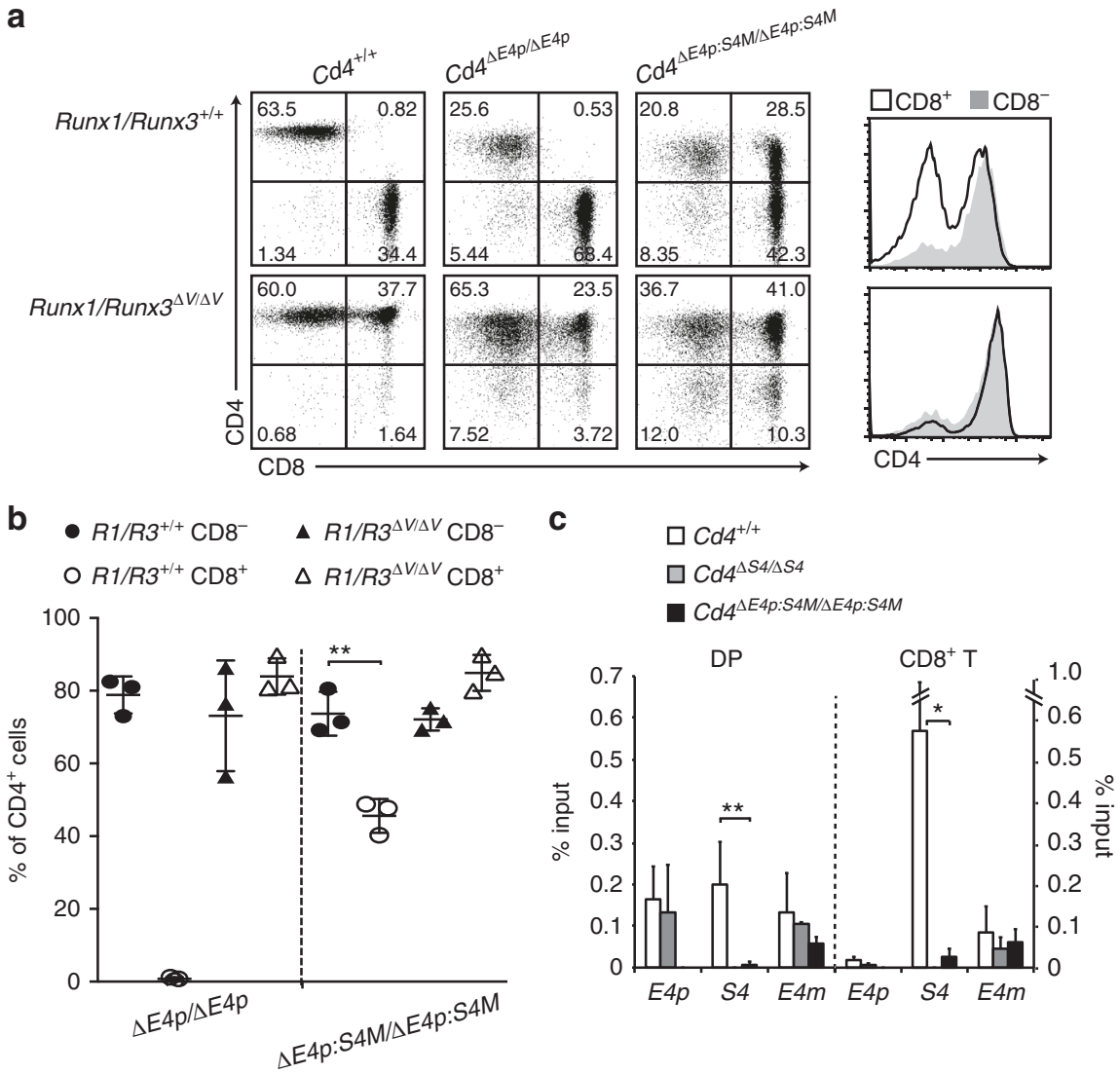

Fig. 6 Involvement of Runx in regulating the helper-lineage dominant activity of E4m. a Dot plots showing CD4 and CD8 expression in lymph node T cells of mice with indicated genotypes. In Runx $1 / R u n \times 3^{\Delta V} / \Delta V$ mice, both Runx1 and Runx3 proteins lack the VWRPY motif, which is essential for interaction with TLE/Groucho co-repressors. Right histograms showing CD4 expression level in CD8- and CD8 ${ }^{+}$lymph node T cells of Cd4 $4 E 4 p: S 4 M / \Delta E 4 p: S 4 M$ mice with (lower) or without (upper) Runx1/Runx $3^{\Delta V / \Delta V}$ mutation. Numbers in quadrants indicate respective cell percentages. One representative result of three independent analyses. b Summary of percentage of CD4 ${ }^{+}$cells in $C D 8^{-}$and CD $8^{+}$lymph node T cells of Cd4 $4 E 4 p / \Delta E 4 p, C d 4^{4 E 4 p: S 4 M / \Delta E 4 p: S 4 M}$ mice with or without Runx1/Runx $3^{\Delta V / \Delta V}$ mutation. Means \pm SD. ${ }^{* *} p<0.01$ (unpaired student $t$ test, two-sided). c Graph showing summary of three independent ChIPqPCR for Runx bindings to E4p, S4, and E4m regions in DP thymocytes (DP) and CD8 ${ }^{+} \mathrm{T}$ cells from Cd4 ${ }^{+} /+, C d 4^{\Delta S 4 / \Delta S 4}$ and Cd4 $4 E 4 p: S 4 M / \Delta E 4 p: S 4 M$ mice. Means \pm SD. ${ }^{\star} p<0.05,{ }^{\star \star} p<0.01$ (One-way ANOVA and Tukey's multiple comparison test)

both proteins are involved in the activation of $E 4 m$ in the $C d 4$ gene and the $P E$ enhancer in the Thpok gene ${ }^{23,27}$. This also suggests that activation of the Thpok gene might involve DNA demethylation. Release of Thpok silencing from $\mathrm{CD}^{+} \mathrm{T}$ cells by an inhibitor for maintenance methyltransferase ${ }^{31}$ and the low Thpok expression level effected by a lack of Tet $2 / 3$ proteins $^{15}$ support the involvement of DNA de-methylation in Thpok activation.

In $B 2 m^{-1-}: C d 4^{\triangle E 4 m / \triangle E 4 m}$ mice, a re-directed $\mathrm{CD} 4^{-} \mathrm{CD} 8^{+}$cell subset is generated in the thymus in part due to impaired ThPOK induction; however, these $\mathrm{CD} 4^{-} \mathrm{CD} 8^{+}$thymocytes failed to expand sufficiently to generate a visible population in the periphery. As these cells are MHC-II restricted, the lack of TCR signaling by loss of CD4 expression could impair their survival capacity $^{32}$. However, previous reports have shown that mice expressing a low level of ThPOK retain re-directed $\mathrm{CD}^{-} \mathrm{CD}^{+}$ MHC-II restricted cells in the periphery ${ }^{16,33}$. It is possible that aberrant CD4 expression kinetics unique to $C d 4^{\triangle E 4 m / \Delta E 4 m}$ mice may generate $\mathrm{CD} 4^{-} \mathrm{CD} 8^{+}$MHC-II restricted cells that acquire IL-7-dependent survival capacity similar to normal MHC-I restricted CD8 SP thymocytes ${ }^{21,22}$ with a low level of IL-7Ra expression. An imbalance between attaining an IL-7-responsive property and inefficient receptor expression might thus inhibit the expansion of $\mathrm{CD}^{-} \mathrm{CD} 8^{+}$MHC-II restricted cells in $C d 4^{\Delta E 4 m / \Delta E 4 m}$ mice.
Our genetic results clearly showed that Runx proteins are necessary for $S 4$-independent repression of $E 4 m$ activity in $\mathrm{CD} 8^{+}$ $\mathrm{T}$ cells. Previous research showed that mutant Runt protein, a counterpart of Runx protein in Drosophila, lacking DNA binding activity by point mutations in the Runt-domain, retains the activity to repress the segment polarity gene engrailed $(e n)^{34}$, indicating that recognition of the Runx-motif is not absolutely necessary for Runx-mediated repression. Once Runx proteins are included into nuclear protein complexes on E4m, TLE/Groucho co-repressor family proteins are likely to be recruited and suppress $E 4 m$ activity in a CD8-lineage-predominant manner. In contrast, although Runx complexes also associate with the E4p enhancer even in the absence of $S 4$, inhibition of E4p activity requires $S 4$ sequences. Thus, the modes of Runx action to repress the two enhancers in the $C d 4$ locus are different, although the VWRPY motif in Runx proteins are shared by two modes. It is possible that protein complexes formed on the $S 4$ region are required specifically to repress transcriptional activators on the E4p enhancer. Alternatively, based on the different position of the two enhancers with respect to $S 4$, topological regulation such as prevention of chromatin looping between E4p and the P4 promoter may require $S 4$-dependent regulation.

An S4-independent regulation of lineage specificity of $E 4 \mathrm{~m}$ activity challenges the previous silencer-based model that has been proposed to explain how lineage-specific $C d 4$ expression is 
a

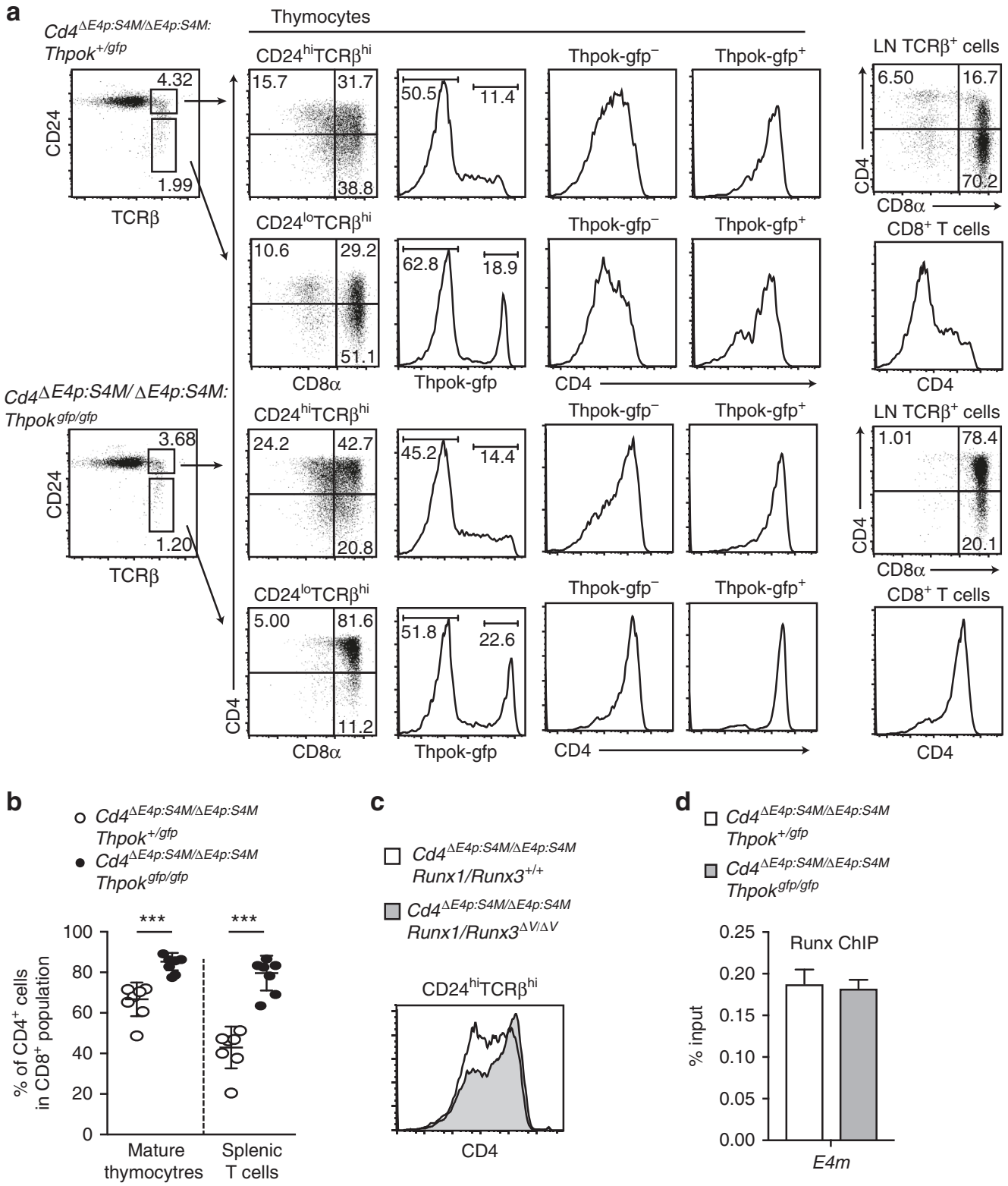

Fig. 7 ThPOK is necessary for Runx-mediated repression of E4m activity. a Flow cytometry analyses for CD4, CD8 and Thpok-gfp expression during T cell development in $C d 4^{\triangle E} 4 \mathrm{p}: S 4 M / \triangle E 4 p: S 4 M$ mice in the presence or absence of ThPOK. Numbers in quadrants, indicated gate, and the region in histograms

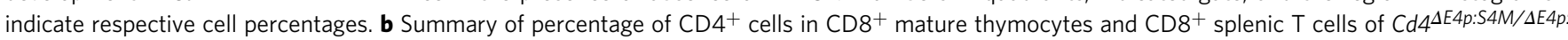
S4M: Thpok ${ }^{+/ g f p}$ and $C d 4 \Delta E 4 p: S 4 M / \Delta E 4 p: S 4 M$ : Thpokgfp/gfp mice. Means \pm SD. ${ }^{\star \star \star} p<0.001$ (unpaired student $t$ test, two-sided). c Histogram showing CD4

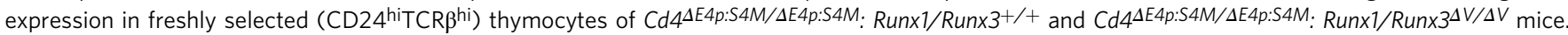
One representative of two independent analyses. $\mathbf{d}$ Graph showing summary of three independent ChIP-qPCR experiments for Runx bindings to E4m in pre-selection DP thymocytes from Cd4 4 E4p:S4M/ $\Delta E 4 p: S 4 M$ : Thpok ${ }^{+/ g f p}$ and $C d 44 E 4 p: S 4 M / \Delta E 4 p: S 4 M$ : Thpokgfp/gfp mice. Means \pm SD

regulated. Association of Runx with E4m in both cytotoxiclineage and helper-lineage cells suggests that Runx-mediated $E 4 m$ repression is likely to be canceled in a helper-lineage-specific manner. ThPOK was shown to antagonize $S 4$ activity to maintain $\mathrm{CD} 4$ expression in helper-lineage cells ${ }^{16,28}$. However, our genetic results indicate that ThPOK instead assists Runx-mediated $E 4 m$ repression. It remains elusive how ThPOK exerts such opposite regulation against $S 4$-dependent and -independent Runx function. Considering that loss of ThPOK expression affects CD4 expression from the $C d 4^{\triangle E 4 p: S 4 M}$ allele in freshly selected thymocytes through MHC-I, it is worth taking into account the possibility that ThPOK expression in early hematopoiesis is involved in later regulation of $E 4 m$ activity. Recruitment of HDACs was proposed to be a possible mechanism by which
ThPOK repress $C d 8$ gene $^{19}$. Along with a higher H3K27ac status at $E 4 m$ region in ThPOK-deficient cells, ThPOK might regulate E4m activity via histone acetylation. Alternatively, considering that ThPOK has been shown to be involved in tethering genomic regions to the nuclear lamina to effect gene repression ${ }^{35}$, it is also possible to ThPOK regulate $C d 4$ expression by altering nuclear positioning of the Cd4 locus during $\mathrm{T}$ cell development. Regardless, our genetic results shed new light on the roles of Runx and ThPOK in $C d 4$ gene regulation by regulating a novel $E 4 m$ enhancer.

\section{Methods}

Generation of mutant Cd4 alleles. In order to generate the target vector for the $C d 4^{E t h}$ allele, we first generated an Eth DNA fragment by overlap PCR. In the Eth 
sequences, core enhancer sequences from the thymic enhancer $(T E)$ and the proximal enhancer $(P E)$ from the Thpok locus were conjugated (Supplementary Fig. 1a). In order to replace E4p with Eth, the Eth DNA fragment was ligated into the SalI site of the target vector (a gift from Mark Chong) that was used to generate the $E 4 p^{\Delta / \Delta}$ mice $^{11}$. Transfection of target vectors into M1 ES cells and identification of ES clone underwent homologous recombination by PCR were performed. During a process of removal of the neomycin resistant gene $\left(n e o^{r}\right)$ by transient transfection of a Cre expression vector, pMC-Cre, ES clones harboring $C d 4^{+/ E t h}$ or $C d 4^{+/ \Delta E 4 p}$ genotype were generated from the into ES clone harboring the $C d 4$ + EthN genotype.

In order to make the target vector for the $C d 4^{\Delta E 4 m}$ allele in which mouse genomic region corresponding to $124835251-124835615$ in NCBI37/mm9 were deleted (Supplementary Fig. 2b), we first amplified the 124834833-1283251 region by PCR to add BglII site at $5^{\prime}$ end and SpeI site at $3^{\prime}$ end. In order to make a longer $3^{\prime}$ homology region, a DNA fragment prepared from the pCassette vector ${ }^{36}$ by SpeI/KpnI digestion was ligated to the $3^{\prime}$ end. A DNA fragment that harbors $5^{\prime}$ homology region, the $n e o^{r}$ gene and the $C d 4$ silencer region was prepared from the pCassette vector by HindII/BglII digestion, and were ligated together with a BglII/ KpnI 3' homology region into the HindIII/KpnI cleaved pUC18 vector. In order to generate ES clones harboring the $C d 4^{+/ \Delta E 4 m}$ and $C d 4^{+/ \Delta E 4 p: \Delta E 4 m}$ genotype, the target vector for the $C d 4^{\Delta E 4 m}$ allele was transfected into ES clone harboring $C d 4^{\Delta S 4 /}$ $\triangle E 4 p$ genotype. After isolating ES clones that underwent homologous recombination, we examined which allele, $C d 4^{+}$or $C d 4^{\Delta E 4 p}$, was targeted by retroviral Cre transduction, followed by PCR that distinguished pattern of recombination between three loxP sites (Supplementary Fig. 2c). ES clones harboring the $C d 4^{+/ \Delta S 4}$ or $C d 4^{+/ \Delta E 4 p: \Delta S 4}$ were similarly generated by transfection of the target vector that remove $S 4$ core region (124835896-124836384 in NCBI37/ $\mathrm{mm} 9$ ) into $\mathrm{Cd} 4^{+/+}$or $\mathrm{Cd} 4^{+/ E t h}$ ES clone, respectively. ES clones harboring the $\mathrm{Cd} 4$ $+/ \triangle E 4 p: S 4 M$ were generated by transfection of the target vector, which was used to target mutation into the Runx sites within the $S 4^{9}$, into the $C d 4^{+/ \Delta E 4 p}$ ES clone.

Mice. Runx $1^{\Delta V}$ mice ${ }^{37}, R u n x 3^{\Delta V}$ mice $^{38}$, Thpokgfp mice ${ }^{16}$ and $B c l 11 b^{F}$ and hypomorphic $B c l 11 b^{m}$ mice $^{23}$ have been described. $\beta 2 \mathrm{~m}$-deficient mice (002087) were purchased from the Jackson laboratory. $C d 4^{+/ \Delta L S}$ mice were generated by crossing $C d 4^{+/ S f l o x}$ mice $^{6}$ (a gift from Dr. D. R. Littman) to the EIIa-Cre mouse (003724) from the Jackson Laboratory. Generation of chimera mice from ES cells by aggregation was performed by the animal facility at RIKEN, IMS. All mice were maintained in the animal facility at the RIKEN IMS, and all animal procedures were in accordance with protocol approved by the institutional Animal Care and Use Committee (IACUC) of RIKEN Yokohama Branch.

Flow cytometry analyses and cell sorting. Single cell suspensions from thymus, spleen and lymph nodes were prepared by mashing tissues through a $70 \mu \mathrm{m}$ cell strainer (BD Bioscience). Single cell suspensions were stained with following antibodies purchased from BD Bioscience or eBiosciences: CD4 (25-0042-81: eBiosciences, RM4-5), CD8a (553035, 563068: BD Biosciences, 53-6.7), CD24 (562563: BD Biosciences, M1/69), CD69 (561931: BD Biosciences, H1.2F3), IL7Ra (17-1271-82: eBiosciences, A7R34) and TCR $\beta$ (47-5961-82: eBiosciences, H57-

597). For intracellular staining of phosphorylated Stat5 (612599: BD Biosciences, 47), cells were stained with cell surface molecules and were stimulated recombinant mouse IL-7 (R\&D) at the indicated time and concentration. After stimulation, cells were fixed and permeabilized with BD Phosflow ${ }^{\text {mex }}$ Lyse/Fix buffer (558049: BD Biosciences) and Perm Buffer III (558050: BD Biosciences). Antibodies were used at a concentration of $2.5 \mu \mathrm{g} \mathrm{ml}^{-1}$. Multi-color flow cytometry analysis was performed using a BD FACSCanto II (BD-Bioscience) and data were analyzed using FlowJo (Tree Star) software. Cell subsets were sorted using a BD FACSAria II (BD Biosciences).

DNA methylation analyses. DNA purified from FACS-sorted cells was subjected to bisulfite reactions with MethylEasy ${ }^{\text {nI }}$ Xceed (Genetic Signatures) according to the manufacturer's protocol. PCR product amplified from this DNA was cloned into the $\mathrm{pCR}^{\mathrm{TM}} \mathrm{II}$ Vector using a TA Cloning ${ }^{\oplus}$ Kit (ThermoFisher scientific) and was sequenced. The following primer set was used for PCR amplification: Cd4R1F, 5'TTGATTTTTTAAAATAGAAAGGTTT- $3^{\prime}$ and Cd4R1R, 5'-AAATATCTAAAATATACCAACCACTAC-3'.

Quantitative RT-PCR. DNase I-treated total RNA was prepared from sorted cells using RNeasy mini kit (QIAGEN) and cDNA was synthesized by SuperScript ${ }^{\text {tix }}$ IV reverse transcriptase (ThermoFisher SCIENTIFIC). Quantitative RT-PCR was performed using QuantStudio ${ }^{\text {mo }} 3$ Real-Time PCR system (Applied Biosystems) with Universal ProbeLibrary (Roche). The following primer sets and probes were used for $C d 4$ and Hprt mRNA quantification: Cd4-F, 5'-TCTGGAACTGCACCGTGAC-3', Cd4-R, 5'-CCGTGATAGCTGTGCTCTGA-3' and UPL \#93. Hprt-F, 5'-TCCTCCTCAGACCGCTTTT-3', Hprt-R, 5'-CCTGGTTCATCATCGCTAATC-3' and UPL \#95.

Chromatin immune-precipitation (ChIP). ChIP-Seq data for Runx/Cbf $\beta$ complexes and Bcl11b binding in total thymocytes were taken from existing deposited data (GEO accession number: GSE90949 and GSE90794, respectively). For analytical ChIP-qPCR, FACS-sorted or MACS (Mitenyi Biotec)-separated 0.5 to $3 \times 10^{7}$ cells were used to prepare chromatin DNA. Cells were cross-linked by incubation in a $1 \%$ of formaldehyde solution for $10 \mathrm{~min}$ with gentle rotation at room temperature. Nuclei were separated and were sonicated using a model XL2000 ultrasonic cell disruptor (MICROSON). Sonicated chromatin was incubated overnight at $4{ }^{\circ} \mathrm{C}$ with anti-Histone H3K27ac (clone D5E4, Cell Signaling Technology), anti-Histone H3K27me3 (clone C36B11), 5 ug of anti-Cbf $\beta$ rabbit polyclonal antibody ${ }^{39}$, anti-Bcl11b antibody (A300-385A, Bethyl Laboratories) or anti-ThPOK polyclonal antibody ${ }^{23}$ that were pre-conjugated with Dynabeads M280 Sheep anti-Rabbit IgG (Thermo Fisher Scientific). After washing beads, immunoprecipitates were eluted from beads into elution buffer. Eluted immunoprecipitates were then incubated at $65^{\circ} \mathrm{C}$ overnight for reverse-crosslinking. Input DNA and ChIP DNA were treated with RNaseA (Thermo Fisher Scientific) and Proteinase K (Thermo Fisher Scientific), and then were purified by ChIP DNA Clean and Concentrator ${ }^{\mathrm{TM}}$ kit (ZYMO RESEARCH). Quantitative PCR was performed using the QuantStudio 3 Real-Time PCR system (Applied Biosystems) with SYBR Green detection system. Primers sequences for quantitative PCR are listed in the Supplementary Table 1.

In vitro T-cell culture. Sorted $\mathrm{CD}^{+}{ }^{+} \mathrm{T}$ cells were labeled with CFSE (ThermoFisher Scientific) and were cultured in custom ordered Dulbecco's Modified Eagle Medium (D-MEM, KOHJIN BIO) supplemented with $10 \%$ heat inactivated FBS (Hyclone). $2.0 \times 10^{5}$ cells were stimulated in 96-well flat-bottomed plate, which was pre-coated with $2 \mu \mathrm{g} \mathrm{ml}{ }^{-1}$ anti-CD3e antibody (553068, BD Bioscience) and $2 \mu \mathrm{g}$ $\mathrm{ml}^{-1}$ soluble anti-CD28 antibody (553295, BD Bioscience), for two days, and were maintained in D-MEM supplemented with $10 \mathrm{ng} \mathrm{ml}^{-1}$ recombinant mouse IL-2 (402-ML, R\&D system) for 2 to 4 days.

Data availability. ChIP-seq data that support the findings of this study have been deposited with accession GSE90949 and GSE90794, respectively. All other relevant data are available from the authors.

Received: 15 May 2018 Accepted: 30 July 2018

Published online: 05 September 2018

\section{References}

1. Zamoyska, R. CD4 and CD8: modulators of T-cell receptor recognition of antigen and of immune responses? Curr. Opin. Immunol. 10, 82-87 (1998).

2. Ellmeier, W., Sawada, S. \& Littman, D. R. The regulation of CD4 and CD8 coreceptor gene expression during T cell development. Annu. Rev. Immunol. 17, 523-554 (1999).

3. Hogquist, K. A. \& Jameson, S. C. The self-obsession of T cells: how TCR signaling thresholds affect fate 'decisions' and effector function. Nat. Immunol. 15, 815-823 (2014)

4. Sawada, S., Scarborough, J. D., Killeen, N. \& Littman, D. R. A lineage-specific transcriptional silencer regulates CD4 gene expression during $\mathrm{T}$ lymphocyte development. Cell 77, 917-929 (1994).

5. Siu, G., Wurster, A. L., Duncan, D. D., Soliman, T. M. \& Hedrick, S. M. A transcriptional silencer controls the developmental expression of the CD4 gene. EMBO J. 13, 3570-3579 (1994).

6. Zou, Y. R. et al. Epigenetic silencing of CD4 in T cells committed to the cytotoxic lineage. Nat. Genet. 29, 332-336 (2001).

7. Leung, R. K. et al. Deletion of the CD4 silencer element supports a stochastic mechanism of thymocyte lineage commitment. Nat. Immunol. 2, 1167-1173 (2001).

8. Taniuchi, I., Ellmeier, W. \& Littman, D. R. The CD4/CD8 lineage choice: new insights into epigenetic regulation during T cell development. Advs Immunol. 83, 55-89 (2004).

9. Taniuchi, I. et al. Differential requirements for Runx proteins in CD4 repression and epigenetic silencing during $\mathrm{T}$ lymphocyte development. Cell 111, 621-633 (2002).

10. Woolf, E. et al. Runx3 and Runx1 are required for CD8 T cell development during thymopoiesis. Proc. Natl Acad. Sci. USA 100, 7731-7736 (2003).

11. Chong, M. M. et al. Epigenetic propagation of CD4 expression is established by the Cd4 proximal enhancer in helper T cells. Genes Dev. 24, 659-669 (2010).

12. Sellars, M. et al. Regulation of DNA methylation dictates Cd4 expression during the development of helper and cytotoxic T cell lineages. Nat. Immunol. 16, 746-754 (2015)

13. He, X. et al. CD4-CD8 lineage commitment is regulated by a silencer element at the ThPOK transcription-factor locus. Immunity 28, 346-358 (2008).

14. Muroi, S., Tanaka, H., Miyamoto, C. \& Taniuchi, I. Cutting edge: fine-tuning of Thpok gene activation by an enhancer in close proximity to its own silencer. J. Immunol. 190, 1397-1401 (2013). 
15. Tsagaratou, A. et al. TET proteins regulate the lineage specification and TCRmediated expansion of iNKT cells. Nat. Immunol. 18, 45-53 (2017).

16. Muroi, S. et al. Cascading suppression of transcriptional silencers by ThPOK seals helper T cell fate. Nat. Immunol. 9, 1113-1121 (2008).

17. Henson, D. M., Chou, C., Sakurai, N. \& Egawa, T. A silencer-proximal intronic region is required for sustained CD4 expression in postselection thymocytes. J. Immunol. 192, 4620-4627 (2014).

18. Shlyueva, D., Stampfel, G. \& Stark, A. Transcriptional enhancers: from properties to genome-wide predictions. Nat. Rev. Genet. 15, 272-286 (2014).

19. Rui, J., Liu, H., Zhu, X., Cui, Y. \& Liu, X. Epigenetic silencing of CD8 genes by ThPOK-mediated deacetylation during CD4 T cell differentiation. J. Immunol. 189, 1380-1390 (2012).

20. Wang, L. et al. The zinc finger transcription factor Zbtb7b represses CD8-lineage gene expression in peripheral CD4 ${ }^{+} \mathrm{T}$ cells. Immunity 29, 876-887 (2008)

21. Park, J. H. et al. Signaling by intrathymic cytokines, not $\mathrm{T}$ cell antigen receptors, specifies CD8 lineage choice and promotes the differentiation of cytotoxic-lineage T cells. Nat. Immunol. 11, 257-264 (2010).

22. Tani-ichi, S. et al. Interleukin-7 receptor controls development and maturation of late stages of thymocyte subpopulations. Proc. Natl Acad. Sci. USA 110, 612-617 (2013).

23. Kojo, S. et al. Priming of lineage-specifying genes by Bcl11b is required for lineage choice in post-selection thymocytes. Nat. Commun. 8, 702 (2017).

24. Levanon, D. et al. Transcriptional repression by AML1 and LEF-1 is mediated by the TLE/Groucho corepressors. Proc. Natl Acad. Sci. USA 95, 11590-11595 (1998).

25. Aronson, B. D., Fisher, A. L., Blechman, K., Caudy, M. \& Gergen, J. P. Groucho-dependent and -independent repression activities of Runt domain proteins. Mol. Cell Biol. 17, 5581-5587 (1997).

26. Seo, W. et al. Roles of VWRPY motif-mediated gene repression by Runx proteins during T-cell development. Immunol. Cell Biol. 90, 827-830 (2012).

27. Kakugawa, K. et al. Essential roles of SATB1 in specifying T lymphocyte subsets. Cell Rep. 19, 1176-1188 (2017).

28. Wildt, K. F. et al. The transcription factor zbtb7b promotes CD4 expression by antagonizing runx-mediated activation of the CD4 silencer. J. Immunol. 179, 4405-4414 (2007).

29. Pastor, W. A., Aravind, L. \& Rao, A. TETonic shift: biological roles of TET proteins in DNA demethylation and transcription. Nat. Rev. Mol. Cell Biol. 14, 341-356 (2013).

30. Tsagaratou, A. et al. Dissecting the dynamic changes of 5hydroxymethylcytosine in T-cell development and differentiation. Proc. Natl Acad. Sci. USA 111, E3306-E3315 (2014).

31. Tanaka, H. et al. Epigenetic Thpok silencing limits the time window to choose CD4(+) helper-lineage fate in the thymus. EMBOJ 32, 1183-1194 (2013)

32. Polic, B., Kunkel, D., Scheffold, A. \& Rajewsky, K. How alpha beta T cells deal with induced TCR alpha ablation. Proc. Natl Acad. Sci. USA 98, 8744-8749 (2001).

33. Egawa, T. \& Littman, D. R. ThPOK acts late in specification of the helper T cell lineage and suppresses Runx-mediated commitment to the cytotoxic T cell lineage. Nat. Immunol. 9, 1131-1139 (2008).

34. Vander Zwan, C. J., Wheeler, J. C., Li, L. H., Tracey, W. D. \& Gergen, J. P. A DNA-binding-independent pathway of repression by the Drosophila Runt protein. Blood. Cells Mol. Dis. 30, 207-222 (2003).

35. Zullo, J. M. et al. DNA sequence-dependent compartmentalization and silencing of chromatin at the nuclear lamina. Cell 149, 1474-1487 (2012).
36. Taniuchi, I., Sunshine, M. J., Festenstein, R. \& Littman, D. R. Evidence for distinct CD4 silencer functions at different stages of thymocyte differentiation. Mol. Cell 10, 1083-1096 (2002).

37. Nishimura, M. et al. VWRPY motif-dependent and -independent roles of AML1/Runxl transcription factor in murine hematopoietic development. Blood 103, 562-570 (2004).

38. Yarmus, M. et al. Groucho/transducin-like Enhancer-of-split (TLE)dependent and -independent transcriptional regulation by Runx3. Proc. Natl Acad. Sci. USA 103, 7384-7389 (2006).

39. Naoe, Y. et al. Repression of interleukin- 4 in T helper type 1 cells by Runx/Cbf beta binding to the Il4 silencer. J. Exp. Med. 204, 1749-1755 (2007).

\section{Acknowledgements}

We thank C. Miyamoto for mouse genotyping, N. Yoza for cell sorting, T. Ishikura for ES cell aggregation and $\mathrm{H}$. Yoshida for providing valuable information for the ATAC-Seq database. This work was supported by JSPS KAKENHI Grant Number 14 F04214 (I.T.) and JP 19390118 (I.T.)

\section{Author contributions}

S.K., N.Y. and M.T. performed phenotypic analyses and ChIP. S.M. generated mouse strains. I.T. designed experiments and wrote the manuscript.

\section{Additional information}

Supplementary Information accompanies this paper at https://doi.org/10.1038/s41467 018-05803-3.

Competing interests: The authors declare no competing interests.

Reprints and permission information is available online at http://npg.nature.com/ reprintsandpermissions/

Publisher's note: Springer Nature remains neutral with regard to jurisdictional claims in published maps and institutional affiliations.

Open Access This article is licensed under a Creative Commons Attribution 4.0 International License, which permits use, sharing, adaptation, distribution and reproduction in any medium or format, as long as you give appropriate credit to the original author(s) and the source, provide a link to the Creative Commons license, and indicate if changes were made. The images or other third party material in this article are included in the article's Creative Commons license, unless indicated otherwise in a credit line to the material. If material is not included in the article's Creative Commons license and your intended use is not permitted by statutory regulation or exceeds the permitted use, you will need to obtain permission directly from the copyright holder. To view a copy of this license, visit http://creativecommons.org/ licenses/by/4.0/

(c) The Author(s) 2018 